\title{
Capability of air exchange rate to predict ventilation of three- dimensional street canyons
}

\author{
Zuzana Kluková ${ }^{1,2, *}$, Štěpán Nosek $^{1}$ and Vladimír Fuka ${ }^{2}$ \\ ${ }^{1}$ Institute of Thermomechanics, Czech Academy of Sciences, Dolejškova 1420/5,182 00 Praha 8, Czech Republic \\ ${ }^{2}$ Department of Atmospheric Physics, Charles University, V Holešovičkách747/2, 18000 Praha 8, Czech Republic
}

\begin{abstract}
As most of the world's population lives in cities, it is critical to understand dispersion processes of pollutants in urban areas. This study focuses on so called air exchange rate (ACH) index, which is frequently used by numerical studies to determine ventilation of street canyons without a simulation of a pollution source. These studies applied the ACH on idealised 2D street canyons, where the ventilation acts only through the one opening - roof top. There are two pertinent questions: i) is the ACH really capable to predict the ventilation of a street canyon without knowing of a pollutant source; and ii) how much the ACH differs between 2D and 3D street canyons? To answer these questions, we performed large-eddy simulations of pollution of complex 3D street canyons from ground-level line sources. We computed ACHs and spatially-average concentrations for three different street canyons and compared these quantities with those from previous studies. Results clearly demonstrate that these quantities strongly depend not only on street-canyon geometry but also on geometry of surrounding buildings. It is also shown that $2 \mathrm{D}$ canyon gives unrealistic result for retention of pollutant within an urban street canyon. The ACH might lead to significant underestimation of the street-canyon ventilation if a source would be outside the canyon.
\end{abstract}

\section{Introduction}

Owing to buildings, people are protected from unfavourable meteorological conditions (e.g., high or low temperatures, precipitation, and high wind speed) in cities. But these same buildings might interfere in the ventilation of the streets [1-3]. Moreover, the accumulation of pollutants in the street network might worsen the indoor air quality of the surrounding buildings [4-6]. This is a typical environmental problem as most of the world's population lives in cities. However, due to complexity of urban canopy flows, which in turn are brought about by the urban morphology [7], the dispersion processes within urban street networks are still not well understood.

It is well known that so called street canyons are the urban pollution hotspots as they restrict the ventilation of pollution from traffic and other anthropogenic activities if the wind is perpendicular to their along axis [8]. Owing to this, the street canyons have been subjected to numerous experimental and numerical studies in order to understand the urban ventilation processes. Numerical methods such as computational fluid dynamics (CFD) are widely used because they do not suffer from similarity criteria as reduced-scale experiments, provide entire velocity and pollution fields, and they are fully controllable in comparison with field experiments. However, CFD models need to be validated before interpreting their results.
Many previous numerical studies [e.g., 9-11] were focused on ventilation of idealised two-dimensional street-canyons using a ventilation index "air exchange rate" $(A C H)$ introduced by Liu et al. [10]. For 2D street canyons the $A C H$ is computed through only one streetcanyon opening, which is the roof top. Due to mass conservation, only the upward vertical velocity fluctuations [9-10] or both the mean and fluctuation part of the upward velocity [11] are integrated across that opening. In cases of 3D street canyons, however, computation of the $A C H$ is not as straightforward as for 2D canyons because other, lateral, openings occur for typical 3D canyon and both the mean and fluctuation parts of all velocities outwards the canyon must be included into the $A C H$ calculations. We are aware of only one study [12] that computed the $A C H$ for 3D through all canyon openings using both the mean and fluctuation parts of all velocities outwards the canyon. However, that study investigated stand-alone street canyons formed by two rectangular blocks of different height (thus also investigated different canyon aspect ratio, $H / B$, where $H$ is the building height and $B$ is the building width), which are not representative of real urban street canyons.

Another pertinent question is whether the $\mathrm{ACH}$ can capture the street-canyon ventilation processes although a pollution source is not simulated. Therefore, the aims of this study are the following: (i) to compute the $A C H$ for three different 3D canyons which are a part of more realistic urban areas; (ii) to compare this $A C H$ with the

*Corresponding author: klukovaz@it.cas.cz 
previous studies; and (iii) to assess the capability of the $A C H$ to predict the street-canyon ventilation by comparing with the spatially-average pollutant concentration of each of the canyon.

\section{Methods}

\subsection{The urban-area model}

The problem was studied on three different street canyons embodied in models of complex built-up areas. The models represented typical geometries of European city centres and were scaled down to 1:400.

The first canyon (A1-R, see Fig. 1a) was part of the model A1 composed of evenly spaced $(B / H=0.8$, where $B$ is the street width and $H$ is the canyon height of $20 \mathrm{~m}$ in full scale) $4 \times 4$ courtyard type buildings with constant length $(L / H=4.8)$ and width $(W / H=2.4)$ and with pitched roofs of constant height $H$. The eaves of the pitched roofs were at height $H_{\mathrm{e}}=0.8 \mathrm{H}$ and hence formed the unit street-canyon aspect ratio (ratio between the canyon height and width, $\left.H_{e} / B=1\right)$.

The second (A2-R) and third (A2-L) canyons (Fig. 1b) were part of the same plan area as model A1 but with buildings of non-uniform heights (varying between $0.8 \mathrm{H}, 1 \mathrm{H}$, and $1.2 \mathrm{H}$, model A2) along each building wall. Each street canyon, however, had the same mean height $H$ as that from the model A1. Note that the right non-uniform canyon (A2-R) represented on average a step-up canyon, whereas the left non-uniform canyon represented, on averaged, a step-down canyon.

\subsection{Large eddy simulations}

For the numerical simulations an open-source model called Extended Large-eddy Microscale Model (ELMM) was chosen and was thoroughly described in [13, 14]. The governing equations in the ELMM were filtered incompressible Navier-Stokes equations:

$$
\frac{\partial \vec{u}}{\partial t}+\nabla \cdot \vec{u} \vec{u}=-\nabla p+\nabla\left(v_{e f f} \nabla \cdot \vec{u}\right),
$$

where $\vec{u}$ is the resolved velocity vector, $p$ is the pressure, and $v_{e f f}$ is the sum of the molecular viscosity $v$ and the eddy viscosity $v_{e}$. ELMM was based on the time-scale eddy viscosity subgrid model [15].

Dispersion of the passive scalar was modelled by the following transport equation:

$$
\frac{\partial c}{\partial t}+\nabla \cdot c \vec{u}=\nabla \cdot\left(\kappa_{e f f} \nabla c\right),
$$

where $c$ is the resolved concentration of passive scalar and $\kappa_{e f f}$ is the effective scalar diffusivity computed from the eddy diffusivity using a constant subgrid Schmidt number $\mathrm{Sc}_{\mathrm{sgs}}=0.7$.

The Navier-Stokes equations were accompinied by the continuity equation

$$
\nabla \cdot \vec{u}=0
$$

The pollution of the canyons was modelled by ground level line sources separately. Each canyon had its own ground-level line source positioned at the centre of the right canyon of the model A1 or A2 (RS in Fig. 1a,b), or at the left canyon of the model A2 (RL in Fig. 1b)

The full description of the numerical setup as well as the parameters of the line sources can be found in [13]. That study also proved the reliability of the LES model through several validation tests. Let us only briefly note that the boundary conditions for the velocity components were chosen periodic in all horizontal directions and free-slip at the top and for the passive scalar they were set to zero concentration for the inflow boundary in the streamwise direction and zero gradient for the outflow boundary in the streamwise direction as well as for the lateral directions.

\subsection{Analysis}

\subsubsection{Air exchange rate}

The air exchange rate $(A C H)$ represents the volumetric air exchange per unit time through investigated streetcanyon openings and it was introduced by [10-12] for 2D street canyons and by [12] for the case of 3D street canyons. Due to the mass continuity, only its positive part, $\mathrm{ACH}_{+}$, is computed for each of the openings as

$$
\begin{gathered}
A C H_{+}=\left.\iint\left[\bar{W}_{+}+w_{+}^{\prime}\right] d S d T\right|_{T} \\
\quad+\left.\iint\left[\bar{V}_{-}+v_{-}^{\prime}\right] d S d T\right|_{R} \\
\quad+\left.\iint\left[\bar{V}_{+}+v_{+}^{\prime}\right] d S d T\right|_{L}
\end{gathered}
$$

where $\bar{W}_{+}$and $\bar{V}_{+}$are the mean positive vertical and lateral velocities, $w_{+}^{\prime}$ and $v_{+}^{\prime}$ are the mean vertical and lateral velocity fluctuations, and $T, R$ and $L$ denotes the top, right and left opening depicted in Fig. 1c. Due to Cartesian coordinate system used in the present study, the velocities at the right opening have negative sign to those for the left opening in order to conserve the "outflow" from the canyon.

For this calculation, full time series of the velocity components are needed. Although a LES model might provide velocity time series, their storage requires huge space on storage drives. From this point of view, we used the mean turbulent quantities as the following:

$$
\begin{gathered}
A C H_{+}=\left.\int\left[\bar{W}_{+}+\frac{1}{2}{\overline{w^{\prime} w^{\prime}}}^{1 / 2}\right] d S\right|_{T} \\
+\left.\int\left[\bar{V}_{-}+\frac{1}{2}{\overline{v^{\prime} v^{\prime}}}^{1 / 2}\right] d S\right|_{R} \\
+\left.\int\left[\bar{V}_{+}+\frac{1}{2}{\overline{v^{\prime} v^{\prime}}}^{1 / 2}\right] d S\right|_{L}
\end{gathered}
$$

To work with non-dimensional quantities, the $\mathrm{ACH}_{+}$ from the equation (5) was normalized by the canyon volume $V$ and the characteristic time $T$ obtaining: 


$$
A C H_{+}^{*}=\frac{T}{V} A C H_{+}=\frac{H}{H L B U_{r e f}} A C H_{+}=\frac{1}{L B U_{r e f}} A C H_{+},
$$

where $U_{\text {ref }}$ is the freestream velocity (at the top of the LES domain), and $L$ and $B$ are the canyon length and width.

\subsubsection{Average pollutant concentration}

To check whether the $A C H+$ predicts the street-canyon ventilation correctly, we compared the $A C H+$ with a spatially-averaged pollutant concentration of each of the investigated canyon computed as

$$
\left\langle C^{*}\right\rangle=\frac{1}{V} \int \overline{C^{*}\left(x_{l}\right)} d V
$$
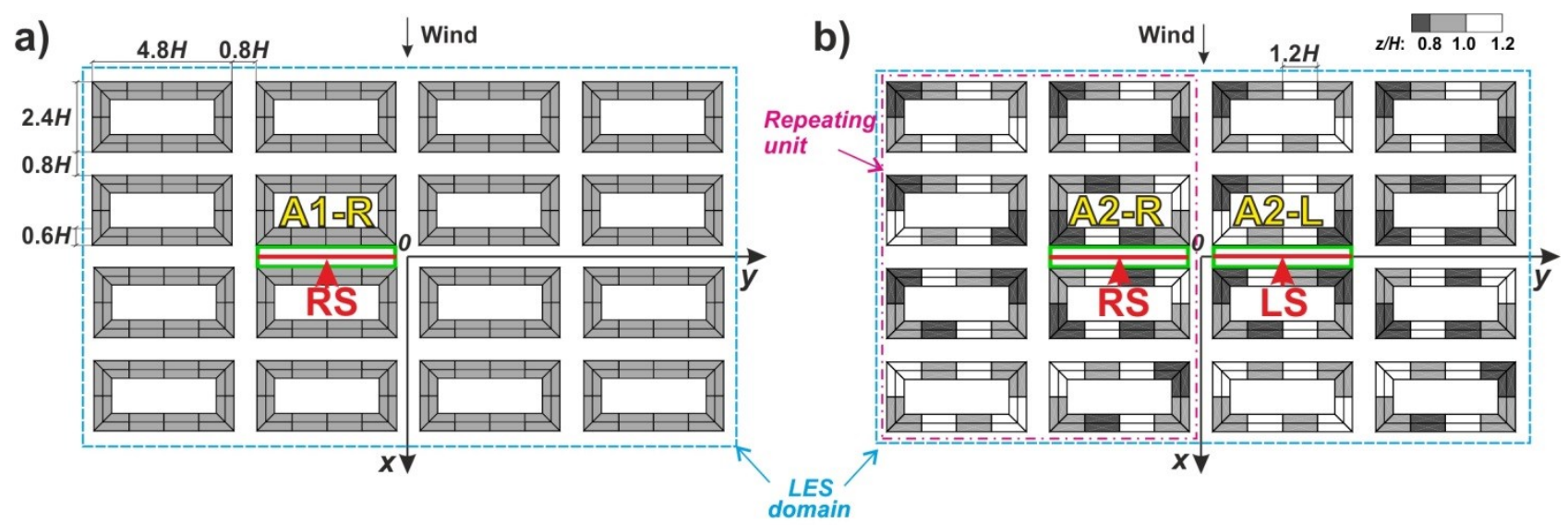

c)

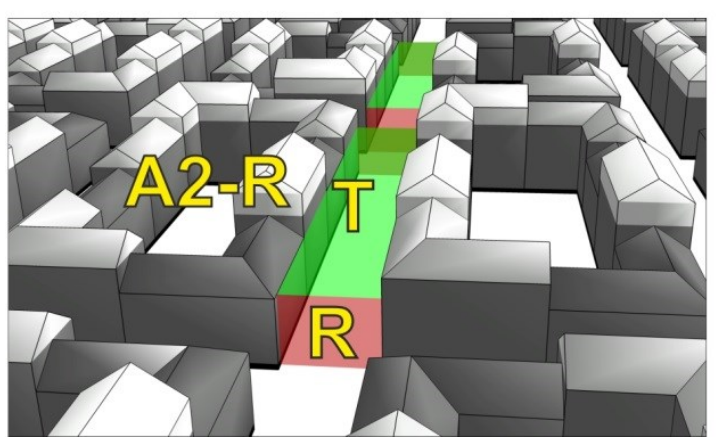

Fig. 1.Schemes of modelled urban areas with the investigated street canyons: a) top view of the uniform urban-array model A1 and b) top view of the non-uniform urban-array model A2, where the green rectangles and red lines mark the investigated canyons (A1-R, A2-R, A2-L) and the pollution source position (RS, LS), respectively. c) 3D view of model A2, where the red and green planes represent the lateral and top openings of the investigated non-uniform canyons, respectively. The grey contour represents the dimensionless height $(\mathrm{z} / \mathrm{H})$ ([13]; partly modified by the present authors).

\section{Results and discussion}

The computed positive air exchange rate for all investigated canyons is presented in Fig. 2 separately for the right $(A C H+R)$, left $(A C H+L)$, and top $(A C H+T)$ opening and also for the sum of these three parts (total air exchange rate $A C H+T O T)$. The $\mathrm{ACH}_{+}$for the particular openings is listed also in Table 1, where it is given relatively (in percent) to the total air exchange rate $A C H+T O T$.

The $\mathrm{ACH}_{+}$through all the openings (and thus for their sum $)$ is the highest $(A C H+T O T=0.088)$ for the right canyon of the non-uniform urban area (A2-R). The

where $c^{*}$ is the instantaneous dimensionless concentration $\left(c^{*}=c U_{\text {ref }} H L / Q\right.$, where $c$ is the instantaneous concentration of passive scalar and $Q$ is the mass flow rate of the source), the overbar denotes the time averaging, and $V=h L B$ denotes the integrated volume.

\subsubsection{Pollutant distribution}

We also computed a relative amount of pollutant as

$$
q_{C}=\left.\int \overline{c^{*}\left(x_{l}\right)} d V\right|_{c a n} /\left.\int \overline{c^{*}\left(x_{l}\right)} d V\right|_{a l l},
$$

where can denotes the volume of the canyon and all means the volume of the entire computation domain. 
the 2D canyon studied by Xie et al. [11] and the 3D canyon studied by Ayo et al. [12]. Both authors computed $\mathrm{ACH}_{+}$using Reynolds-Averaged NavierStokes (RANS) models. The $A C H_{+}$predicted by Xie et al. for $2 \mathrm{D}$ canyon is slightly higher $(A C H+T O T=$ $0.0075)$ than those predicted by the current LES model for the presented $3 \mathrm{D}$ canyons $\mathrm{A} 1-\mathrm{R}(A C H+T O T=$ $0.068)$ and A2-L $(A C H+T O T=0.068)$, and slightly lower than that of the canyon A2-R $(A C H+T O T=$ 0.088). This confirms that the non-uniform step-up canyon (A2-R) has the best ventilation performance among the presented canyons. On the other hand, the total air exchange rate through the 3D canyon investigated by Ayo et al. [12] is significantly higher than those presented by this study.

It is important to note, however, that the 3D street canyon from Ayo et al. [12] was only formed by two isolated buildings (rectangular barns) without any surrounding buildings. Thus, it is expected that the ventilation of such street-canyon configuration will be higher than for a configuration with a built-up area similar to our cases.

Table 1. Air exchange rate for the particular opening $(A C H+R$, $A C H+L, A C H+T$ ) of investigated canyons (A1-R, A2-R, A2-

$\mathrm{L})$ given relatively to the total air exchange rate $A C H+T O T$

\begin{tabular}{|c|ccc|}
\hline & $\begin{array}{c}A C H+R \\
{[\% \text { ACH+TOT }]}\end{array}$ & $\begin{array}{c}A C H+L \\
{[\% A C H+T O T]}\end{array}$ & $\begin{array}{c}A C H+T \\
{[\% A C H+T O T]}\end{array}$ \\
\hline A1-R & 6.8 & 7.8 & 85.4 \\
A2-R & 9.4 & 9.8 & 80.8 \\
A2-L & 8.0 & 6.5 & 85.5 \\
\hline
\end{tabular}

The spatially-average pollutant concentrations $\left\langle C^{*}\right\rangle$ for each of the investigated canyons are plotted in Fig. 3. To compare the results with previous studies, we also present $\left\langle C^{*}\right\rangle$ computed by Liu et al. [11] for 2D canyon and by Michioka et al. [16] for 3D canyon, both using an LES model. Because of the non-uniformity of the pitched roofs in our study, $\left\langle C^{*}\right\rangle$ were computed for two different volumes $V=h_{1} L B=0.6 H L B$, where height $h_{l}=0.6 \mathrm{H}$ corresponded to the height of the lowest building's eaves in the case of non-uniform canyons, and $V=h_{2} L B=0.8 H L B$, where $h_{2}=0.8 H$ corresponded to the mean height of the eaves. The height $h_{2}$ formed the volume of unit aspect ratio (ratio between the canyon's height and canyon's width, $h / B=1)$, and hence the results were more comparable with those from Liu and Michioka in that case. However, in cases of non-uniform canyons, $\left\langle C^{*}\right\rangle$ might be reduced or increased due to the roof-height non-uniformity which might produce flow passages or blockages for incoming "fresh" air. Table 2 presents the relative pollution mass $q_{C}$ of all investigated canyons and that of Liu.

The results clearly show that the average concentration $\left\langle C^{*}\right\rangle$ (Fig. 3) as well as the relative amount of pollutant in the canyon $q_{C}$ are the lowest for the right canyon of the non-uniform urban area (A2-R). These both results are in accordance with primary hypothesis that higher air exchange rate results in better street-canyon ventilation. However, the air exchange rate does not take into account either a pollutant source or the source position. In our previous study [13] we clearly demonstrated that the position of the source might be critical for street-canyon pollution. In that study we simulated the pollution of the identical canyons but with a ground-level line source running from one canyon to another across the intersections. Appreciable pollutant transports were observed not only from the intersections but also from the canyon A2-R to the canyon A2-L through the lateral openings, and $\left\langle C^{*}\right\rangle$ were thus significantly higher in A2-L than in the current A2-L canyon polluted just from the source LS. We observed the same phenomena for the uniform canyon (A1-R) but in lesser extent which resulted in lower $\left\langle C^{*}\right\rangle$ than in the case of the A2-L canyon. Because the air exchange rate shows the same for both (A2-R and A2-L) canyons, the concept of $\mathrm{ACH}$ might lead in such cases to misleading interpretation of street-canyon ventilation.

The comparison of $\left\langle C^{*}\right\rangle$ with previous studies demonstrates that $\left\langle C^{*}\right\rangle$ is strongly dependent on the canyon geometry. The idealised $2 \mathrm{D}$ case from Liu et al. shows, as expected, the highest $\left\langle C^{*}\right\rangle$ because the pollutant is removed, unrealistically, only through the top opening. This is also confirmed by the relative amount of pollutant $q_{C}$ (Table 2) for which the 2D canyon retains $97 \%$ of pollutant contrary to $38 \%$ of the worse ventilated $3 \mathrm{D}$ canyons (A21-R and A2-L in Table 2). The 3D canyon from Michioka et al. has $\left\langle C^{*}\right\rangle$ within the values of all the canyons presented by our study (A1-R, A2-R and A2-L). It is noteworthy that the canyon from Michioka et al. was formed by rectangular blocks (thus without pitched roofs) of length $L=4 H$ (shorter than in our study, $L=4.8 H$ ) and this canyon was surrounded by canyons of the same geometry, which were evenly spaced along the streamwise and spanwise direction and hence forming different street network topology than in our cases. However, despite the shorter length and width of the buildings, the canyon from Michioka et al. has $\left\langle C^{*}\right\rangle$ appreciably higher that of the right non-uniform canyon. This confirms that the averaged step-up canyon performs the best ventilation among investigated and compared canyons.

Table 2. Relative amount of pollutant $q_{C}$ inside all investigated canyons (A1-R, A2-R, A2-L) and the canyon of Liu et al. [10].

\begin{tabular}{|l|c|}
\hline & $q_{C}[\%]$ \\
\hline A1-R & 38.3 \\
A2-R & 28.4 \\
A2-L & 38.8 \\
Liu2005_2D & 97.1 \\
\hline
\end{tabular}




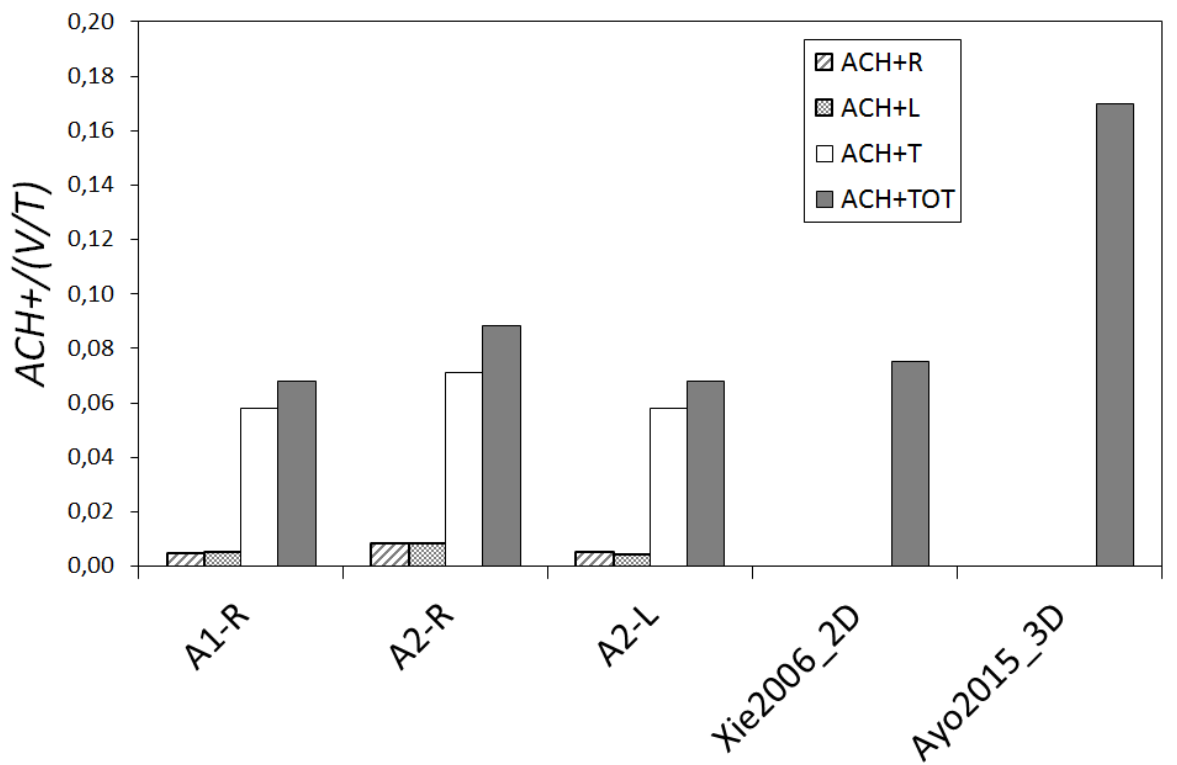

Fig. 2. Total $(A C H+T O T)$ and separate $(A C H+R, A C H+L, A C H+T)$ air exchange range for the investigated canyons and their individual openings (right R, left L, top T). The study of Xie et al. [11] on 2D street canyon and study of Ayo et al. [12] on 3D standalone street canyon is added for comparison.

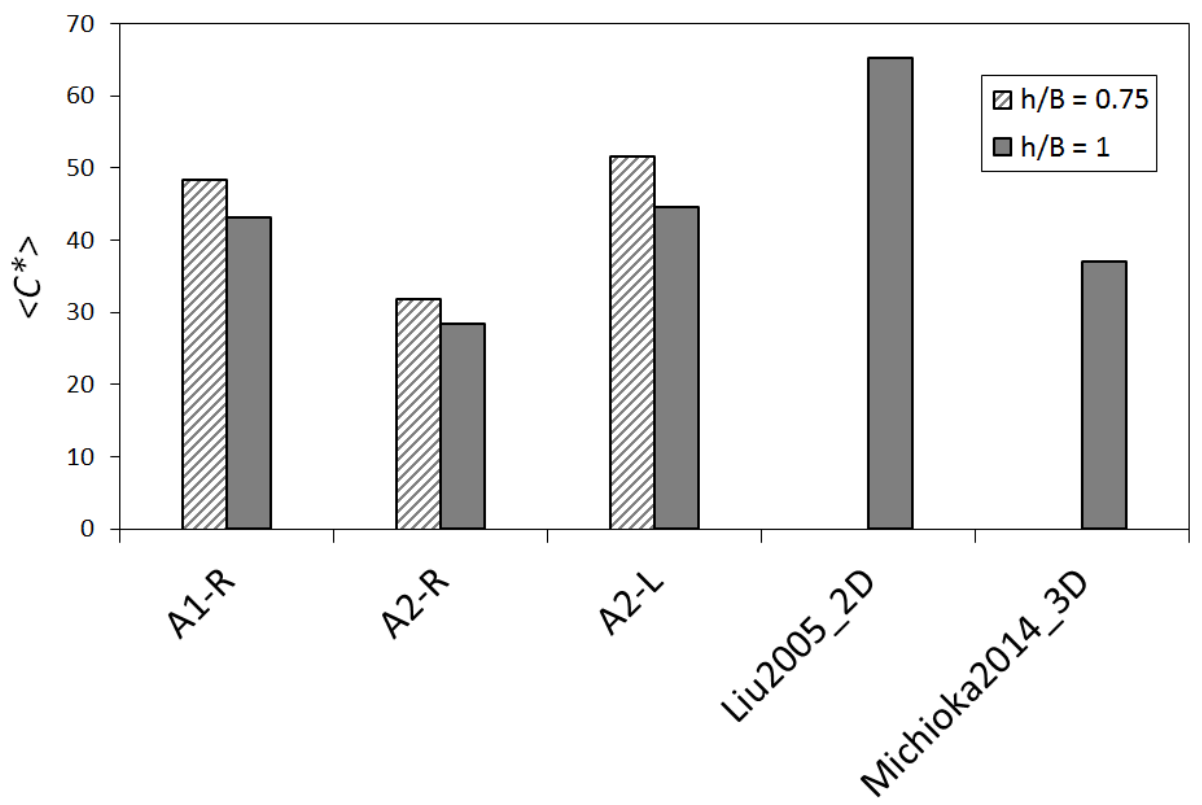

Fig. 3. Spatially-averaged pollutant concentration for all investigated canyons. In the canyons A1-R, A2-R, and A2-L the aspect ratio $h / B=0.75$ and $h / B=1$ corresponds to averaging over the volume $V=h_{1} L B=0.6 H L B$ and $V=h_{2} L B=0.8 H L B$, respectively. The study of Liu et al. [10] on 2D street canyon and study of Michioka et al. [16] on 3D stand-alone street canyon is added for comparison.

\section{Conclusions}

The main aim of this study was to evaluate capability of air exchange rate, $A C H$, to predict the street-canyon ventilation for $3 \mathrm{D}$ canyons. The study was performed on three different 3D canyons (one with uniform and two with non-uniform roof heights) by employing an LES model.

It was found that the $A C H$ is the highest for the nonuniform canyon with local step-up roof arrangements at the lateral ends (A2-R), which caused higher outflows through these lateral openings. This canyon reported also the lowest average concentration $\left\langle C^{*}\right\rangle$ and relative amount of pollutant in the canyon $q_{C}$, which confirmed the primary hypothesis that higher $A C H$ leads to better street-canyon ventilation and thus lower concentration. However, the $\mathrm{ACH}$ cannot be used for prediction of street-canyon ventilation in cases for which pollution sources is also outside the canyon since the concept of the $A C H$ takes into account only the velocity 
components outwards the canyon and did not reflect the potential inflow of pollutants.

$A C H,<C^{*}>$, and $q_{C}$ from this study were compared also with results from previous studies. The main conclusion is that these quantities strongly depend not only on street-canyon geometry but also on geometry of surrounding buildings and the source position. In other words, the street-canyon ventilation cannot be estimated purely from the fundamental canyon parameters such as the building average height, building length, and street width.

The study was supported by the Charles University, project GA UK No. 1583217.

\section{References}

1. W.C. Cheng, C-H Liu, D.Y.C. Leung. Computational formulation for the evaluation of street canyon ventilation and pollutant removal performance. Atmos. Environ. 42, 9041-9051 (2008)

2. S.H.L. Yim, J.C.H. Fung, A.K.H. Lau, S.C. Kot. Air ventilation impacts of the "wall effect" resulting from the alignment of high-rise buildings. Atmos. Environ. 43, 4982-4994 (2009)

3. J.O.P. Cheung, C-H Liu. CFD simulations of natural ventilation behaviour in high-rise buildings in regular and staggered arrangements at various spacings. Energy Build. 43, 1149-1158 (2011)

4. B. Givoni. Climate considerations in building and urban design. New York: Van Nostrand Reinhold (1998)

5. F. Allard, C. Ghiaus. Natural ventilation in the urban environment assessment and design. London: Earthscan (2005)

6. W. Ji, B. Zhao. Numerical study of the effects of trees on the outdoor particle concentration distributions. Build. Simul. 7, 417-427 (2014)
7. C.S.B. Grimmond, T.R. Oke. Aerodynamic properties of urban areas derived from analysis of surface form. J. Appl. Meteorol. 38(9), 1262-1292 (1999)

8. J. Fenger. Urban air quality. Atmos. Environ. 33, 4877-4900 (1999)

9. X-X. Li, C-H. Liu, D.Y.C. Leung. Development of a $k-\varepsilon$ model for the determination of air exchange rates for street canyons. Atmos. Environ. 39, 72857296 (2005)

10. C-H Liu, D.Y.C. Leung, M.C. Barth. On the prediction of air and pollutant exchange rates in street canyons of different aspect ratios using largeeddy simulation. Atmos. Environ. 39, 1567-1574 (2005)

11. X. Xie, C-H. Liu, D.Y.C. Leung, M.K.H. Leung. Characteristics of air exchange in a street canyon with ground heating. Atmos. Environ. 40, 63966409 (2006)

12. S.A. Ayo, N. Mohd-Ghazall, S. Mansor. Outdoor ventilation performance of various configurations of a layout of the adjacent buildings under isothermal conditions. Build. Simul. 8, 81-98/2015)

13. Š. Nosek, V. Fuka, L. Kukačka, Z. Kluková, Z. Jaňour. Street canyon pollution with respect to urban-array complexity: The role of lateral and mean pollution fluxes. Build. Environ. 138, 221-234 (2018)

14. V. Fuka, Z.T. Xie, I.P. Castro, P. Hayden, M. Carpentieri, A.G. Robins. Scalar fluxes near a tall building in an aligned array of rectangular buildings. Bound.-Layer Meteorol. 1-24 (2017)

15. M. Inagaki, T. Kondoh, Y. Nagano. A mixed-timescale SGS model with fixed model parameters for practical LES. J. Fluid.Eng. 127, 1-13 (2005)

16. T. Michioka, H. Takimoto, A. Sato. Large-eddy simulation of pollutant removal from a threedimensional street canyon. Bound.-Layer. Meteorol. 150, 259-275 (2014) 